\title{
TELEOLOGÍA Y TEOLOGÍA EN LA FENOMENOLOGÍA DEL JOVEN DERRIDA
}

\author{
TELEOLOGY AND THEOLOGY IN \\ PHENOMENOLOGY OF YOUNG DERRIDA
}

\author{
Jimmy Hernández Marcelo ${ }^{1}$ \\ Universidad de Salamanca, España \\ Laboratorio de Ontología, Universidad de Turín, Italia
}

\begin{abstract}
Resumen: El presente estudio tiene como objetivo fundamental exponer la fenomenología del joven Derrida (1953-1967) en relación con el problema de Dios como telos universal. A partir del problema de la génesis, Derrida interpreta el proyecto filosófico de Husserl como un pensamiento revolucionario que intenta superar los problemas clásicos de la filosofía. En esta perspectiva surgen los primeros temas filosóficos del pensamiento derridiano, tales como la fenomenología de la escritura, la tradicionalidad y una embrionaria filosofía del documento. En este itinerario, Derrida se encuentra con la propuesta de Husserl sobre la divinidad trascendental que proporcionaría unidad al telos y al arche. Esto le servirá de instrumento para introducir una idea recurrente en la filosofía del joven Derrida: la unidad entre idealidad e historicidad.
\end{abstract}

Descriptores: Joven Derrida, Fenomenología, Teología, Teleología, Génesis

Abstract: The present study attempts to expose the Phenomenology of the young Derrida (1953-1967) in relation to the problem of God as a universal telos. From the problem of the genesis, Derrida interprets the philosophical project of Husserl as a revolutionary thinking that tries to overcome the classical problems of philosophy. In this perspective, the first philosophical themes of Derridian thought emerge, such as the phenomenology of writing, traditionality, and an embryonic philosophy of document. In this itinerary, Derrida meets Husserl's proposal on the transcendental divinity that would provide unity to the telos and the arche. This will serve as an instrument to introduce a recurrent idea in the philosophy of the young Derrida: the unity between ideality and historicity

Keywords: Young Derrida, Phenomenology, Theology, Teleology, Genesis

\footnotetext{
${ }^{1}$ E-mail: jimhermar@usal.es ¡immy.hernandezmarce@edu.unito.it
} 


\section{INTRODUCCIÓN}

La deconstrucción de Jacques Derrida es, sin duda, una de las formas de hacer filosofía más influyente entre los partidarios del posmodernismo. Es cierto que a través de las muy ingeniosas argumentaciones, Derrida supo guiar a sus lectores hacia terrenos dejados al margen en la reflexión filosófica. De allí que su filosofía se mueve siempre en los márgenes y sea, a la vez, una filosofía de los márgenes. Sin embargo, la comprensión del origen, sentido y alcance de su método -si bien es cierto que el propio Derrida rehúsa utilizar el nombre de método para su filosofía y prefiere denominarlo estrategia- es una tarea que todavía no se ha llevado a cabo, al menos, de manera rigurosa en lengua castellana. El período en el que se desarrolla, evoluciona y finalmente se consolida el método empieza en 1953 -durante el proceso de investigación, redacción y culminación de su Memoria de fin de estudios en la Escuela Normal Superior de París-y se extiende hasta 1967, momento en el que elabora un escrito sobre Husserl -inédito hasta el momento. Se conserva de este texto perdido una descripción de su contenido en la que aparecen integrados, por un lado, el artículo de 1966 sobre la fenomenología

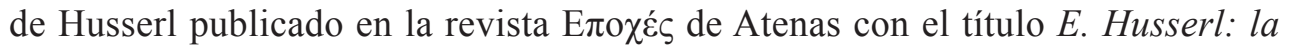
fenomenología y la clausura de la metafísica (Derrida, 1966) ${ }^{2} \mathrm{y}$, por otro lado, la famosa obra La vox y el fenómeno (Derrida, 1967b).

Por nuestra parte, hemos denominado periodo de juventud de Derrida -o simplemente el joven Derrida- a los años que van de 1953 a 1967. Es evidente que este espacio de tiempo ha sido descuidado por los especialistas en lengua castellana y resulta paradójico que se haya dejado al margen, precisamente una etapa que resulta imprescindible conocer y comprender para acceder al significado profundo de la filosofía derridiana. En otras lenguas existen estudios ya clásicos sobre el tema, entre los principales cabe mencionar el libro de Paola Marrati La Genèse et la trace: Derrida lecteur de Husserl et Heidegger (MarratiGuénoun, 1998), el de Leonard Lawlor Derrida and Husserl: The basic problem of phenomenology (Lawlor, 2002). Asimismo, los más recientes son el de Edward Baring The young Derrida and French philosophy, 1945-1968 (Baring, 2011) y el de Françoise Dastur Déconstruction et Phénoménologie. Derrida en débat avec Husserl et Heidegger (Dastur, 2016). Por otro lado, tenemos algunos artículo de celebres fenomenólogos y conocedores del pensamiento de Derrida como Rudolf Bernet (1986, 1990), Natalie Depraz (2000), Daniel Giovannangeli (2013), Raoul Moati (2013) y Vittorio Perego (2016).

\footnotetext{
${ }^{2}$ La versión francesa de este artículo no se publicó sino hasta el año 2000 en la revista Alter, en un número especial dedicado a Derrida y la fenomenología (Derrida, 2000). En 2018 se ha publicado la versión castellana de este importante escrito fenomenológico (Derrida, 2018).
} 
A finales del 2015 apareció en Salamanca la traducción castellana de la Memoria de Derrida de 1953-1954 El problema de la génesis en la filosofía de Husserl (Derrida, 2015). El artículo de 1966, La fenomenología y la clausura de la metafísica, ha sido traducido por nosotros y publicado en 2018 en la revista Investigaciones fenomenológicas, dirigida diligentemente desde hace algunos años por el profesor Javier San Martín. En este mismo número de la revista se incluye un estudio introductorio sobre el joven Derrida y su relación con el movimiento fenomenológico en Francia titulado Derrida en la escuela de la fenomenología (Hernandez Marcelo, 2018b). Asimismo, se ha publicado también una monografía de nuestra autoría sobre este tema (Hernandez Marcelo, 2018a).

Después de hacer una presentación del contexto en el que se desarrollará la presente investigación, nos centraremos en el tema. Sabemos que el período de juventud es un momento de fuerte influencia husserliana, de tal forma que sus primeros escritos o bien están consagrados al estudio de la fenomenología, o bien son aplicaciones, defensas o profundizaciones de la filosofía de Husserl. Podríamos decir, sin temor a equivocarnos, que la primera y más duradera influencia del joven Derrida es Husserl y la fenomenología entendida como una filosofía de la apertura. En el interior de estas primeras investigaciones fenomenológicas vemos aparecer también el problema de Dios. Si bien es cierto, no hay ningún texto que se dedique exclusivamente al tema; no obstante, emergen a lo largo de este período ideas sobre la teleología y la teología como profundización de los comentarios del propio Husserl. Sobre la posibilidad de desarrollar una fenomenología de lo divino en general o del fenómeno religioso, ya uno de los primeros discípulo de Husserl, Jean Héring, sostenía en 1926 que "el mismo Husserl cree -contrariamente a Scheler- en la posibilidad de desarrollar dos ciencias independientes, pero complementarias, que conduzcan a la teología: una filosofía religiosa y una metafísica teleológica" (Héring, 1926, p. 101). El joven Derrida se decantará por explorar la segunda de las vías abiertas por el padre de la fenomenología.

Esta temática se materializará en el año académico 1962-1963 cuando siendo profesor asistente de filosofía general y lógica en la Sorbona (1960-1964) organice cuatro sesiones en un curso denominado Phénoménologie, téléologie, théologie: le Dieu de Husserl (Derrida, 2013, p. 11). En lo que concierne a este estudio, intentaremos hacer explícita la concepción que el joven Derrida tenía de Dios a partir del análisis de sus investigaciones fenomenológicas. En este sentido, nuestra fuente principal serán todas las obras publicadas en el período de juventud y, en concreto, las que se consagran estrictamente a la fenomenología. Por ello mismo, este ensayo podría muy bien llevar el título de Investigaciones fenomenológicas sobre el problema de Dios en el joven Derrida. 


\section{Del PRoBlema de LA GÉNESIS A LA TELEOLOGÍA INTENCIONAL}

Como primer puerto en nuestra investigación, navegamos hacia el primer escrito de Derrida, elaborado como Memoria de estudios superiores -equivalente al grado actual de Máster- para la prestigiosa Escuela Normal de París. Este trabajo estuvo consagrado al estudio del problema de la génesis en la filosofía de Husserl (Derrida, 1990). Para realizar la investigación, Derrida pedirá la colaboración de Maurice de Gandillac. En enero de 1954 Maurice de Gandillac envía una carta de recomendación al padre Van Breda para que autorice a Derrida la consulta de los manuscritos de Husserl conservados en Lovaina. En marzo de aquel mismo año el joven estudiante pasará varias semanas en los Archivos Husserl. En esta ciudad entabla amistad con Rudolf Boehm y ambos suelen hacer paseos por la ciudad y discuten sobre Husserl, Sartre, Merleau-Ponty y Heidegger. Durante su estancia en la ciudad que custodia el legado de Husserl, Derrida conocerá de primera mano el texto El origen de la geometría (Peeters, 2010, p. 91).

Derrida en su Memoria muestra un gran dominio de la filosofía husserliana, pues no realiza una exposición sobre una obra o un período, sino que asume el reto de descifrar la totalidad del proyecto filosófico del padre de la fenomenología. Su propuesta quiere sustituir la fenomenología a la francesa -desarrollada por Sartre y Merleau-Ponty- una fenomenología vuelta hacia las ciencias (Peeters, 2010, p. 90). No obstante, Derrida se siente decepcionado de la ausencia de respuesta frente a su primera obra de envergadura (Peeters, 2010, p. 92). Ni Althusser ni Foucault se interesan por el texto, el único al que le parece digno de atención fue Jean Hyppolite, quien recomienda a Derrida preparar su publicación. Esta no llegaría sino treinta y siete años más tarde, en 1990. Según explica el profesor Maurizio Ferraris, gran conocer de la obra de Derrida - consciente de la deuda que su teoría sobre los objetos sociales tiene con la filosofía de la escritura de Derrida- ${ }^{3}$ estas investigaciones le permiten alcanzar tres argumentos fundamentales de su filosofía: 1) la esencia de la iterabilidad es la repetitividad; 2) la repetitividad aparece esencialmente relacionada con fenómenos como el de la escritura y la huella en general; 3) la desaparición del sujeto representa una condición necesaria para la configuración de la idealidad como iterabilidad (Ferraris, 2006, p. 62).

En este escrito el tema de la teleología está vinculado al problema de la génesis. De hecho, para Derrida la noción de génesis otorga unidad a toda la filosofía de Husserl (Derrida, 1990, p. 2) y en el intento de comprender el

\footnotetext{
${ }^{3}$ Maurizio Ferraris nunca ha dejado de reconocer la influencia de la filosofía derridiana en el desarrollo de su propia propuesta filosófica. En concreto, hablamos del surgimiento de la Documentalidad como continuación de la Gramatología (Ferraris, 2009). No obstante, Ferraris ha manifestado siempre la convicción de superar y corregir la reducción del mundo a las interpretaciones. Sobre la reconstrucción de la deconstrucción, véase (Ferraris, 2010).
} 
problema del origen -tanto empírico como trascendental-, la fenomenología es dirigida inexorablemente hacia una filosofía de la historia (Derrida, 1990, p. 4), es decir, hacia una teleología en la que se comprenda el sentido en la historia como realización. Las tres primeras partes las consagra íntegramente el problema de la génesis: (1) los dilemas de la génesis psicológica, (2) la neutralización de la génesis y (3) el tema fenomenológico de la génesis. Al final, en la cuarta parte, se detiene en la teleología intencional como fundamento de todos los momentos anteriores de la fenomenología (Derrida, 1990, p. 39).

Derrida está convencido de que la reducción de la génesis empírica -o psicológica- ha sido siempre provisional. Así lo demuestra mediante un estudio detallado tanto de la etapa pre-fenomenológica de Husserl como de la denominada genética, en donde la génesis vuelve al corazón mismo de la fenomenología. Una primera idea que debemos señalar es que para Derrida la reducción significa neutralización y nunca, y en ningún sentido, eliminación o negación (Derrida, 1990, p. 132). Aunque Husserl haya suspendido el mundo ante-predicativo, en ningún caso quedó fuera de su horizonte de comprensión. Para Derrida la fenomenología francesa ha asumido esta negación del mundo ante-predicativo como la aplicación de la reducción husserliana. Por tanto, el mayor malentendido en la interpretación de la fenomenología habría consistido, precisamente, en considerar que la reducción conducía a la destrucción de la facticidad empírica (Derrida, 1990, p. 28, nota 43).

La consideración estática de las idealidades (Investigaciones lógicas) y la fenomenología de la conciencia constituyente (Ideas I), según entiende Derrida, preparan el camino para la fenomenología genética (Derrida, 1990, p. 107). Sin embargo, la génesis había sido el punto de partida de Husserl, recuérdese que el tema de Filosofía de la aritmética era ya el origen de los conceptos matemáticos y, en aquel momento, Husserl se decantaba por una posición que Derrida ha denominado "psicologismo intencional" (Derrida, 1990, p. 55). Esto quiere decir que el tema de la génesis fue puesto entre paréntesis -es decir, reducido- y retomado en el momento en el que la pregunta por la génesis adquiere sentido dentro de una filosofía fenomenológica. Aquí no se ignora la eidética, sino que la profundiza mostrando la relación entre el hecho y la esencia, entre lo empírico y lo trascendental, pues Husserl es consciente de que la fenomenología debe ser también genética para poder respetar la temporalidad de la vivencia originaria (Derrida, 1990, p. 14). Husserl ve ahora necesaria una mirada hacia el momento anterior a toda eidética y se dirige hacia la esfera de la existencia ante-predicatica del mundo de la vida y de la intersubjetividad trascendental. En este momento retoma sus investigaciones sobre lógica (Lógica formal y lógica trascendental) y se aproxima a una recuperación filosófica de la historia que permita una reconciliación entre la fenomenología y las ciencias mundanas (Crisis). 
Derrida entiende perfectamente que la fenomenología también tiene como tarea elucidar el problema de la génesis en su constitución temporal mundana, esto es, en la historia tal como es presentado en Crisis y en El origen de la geometría. Toda verdad histórica posee en su interior un sentido intencional y trascendental de todo pasado humano, de todo acto y de toda producción de la conciencia, los cuales son accesibles si se le sabe interrogar (Derrida, 1990, p. 262). Desde el punto de vista trascendental, el sentido de la historia (génesis) es constituido también por un ego trascendental (telos). De este modo, el problema del origen lleva a una filosofía de la historia, como dijimos al principio.

Derrida explica que el estudio sobre la génesis trascendental aparece en Experiencia y juicio. A pesar de inaugurar las investigaciones genéticas, la temporalidad aquí expuesta está ya constituida como lo estaba en Lecciones sobre la conciencia interna del tiempo y como en Ideas I (Derrida, 1990, p. 203). Husserl no daría cuenta de la génesis histórica del mundo de la vida y, por tanto, en Experiencia y juicio todavía se mantendría la génesis entre paréntesis (Derrida, 1990, p. 206).

Por su parte, en Lógica formal y lógica trascendental la temporalidad también está ausente (Derrida, 1990, p. 212) y Meditaciones cartesianas nos anunciaría la nueva orientación fenomenológica. Las tres primeras Meditaciones vuelven sobre los mismos problemas de Investigaciones lógicas, Ideas I y Experiencias y juicio. La cuarta Meditación nos conduce a la contradicción de la génesis activa del yo. Sin embargo, el problema de la constitución de este ego, nos conduciría a los problemas constitutivos en general (Derrida, 1990, p. 224). Finalmente, la quinta Meditación introduce la noción de génesis pasiva, lo cual nos remitiría a la necesidad de una filosofía de la historia. De este modo, se comprende mejor por qué a partir de 1925 la teleología aparece como una temática filosófica en la fenomenología (Derrida, 1990, p. 241). Aquí el peligro sería el que la teleología se mostrase como unidad de sentido ya constituido, puesto que la síntesis pasiva introduce el mundo constituido en la esfera trascendental y hace necesario una elucidación de la historia. Husserl se introduce, entonces, en la constitución temporal del ego y la constitución temporal de la intersubjetividad (Derrida, 1990, p. 247). Es ahora cuando Crisis hace su aparición como una mediación entre el yo trascendental puro y la encarnación empírica de la idea teleológica (Derrida, 1990, p. 249).

En Crisis la definición empírica de Europa presenta la filosofía como llevada en el corazón de ciertos hombres y como fenómenos histórico originario (Derrida, 1990, p. 252). Esta pondría en evidencia una contradicción, pues se podría reducir la idea a un hecho. Ahora bien, para resolver esta nueva aporía, la fenomenología exigirá un giro radical que lleva a salir de la esfera eidética (Derrida, 1990, p. 256). El origen de la geometría expone magistralmente la superación de esta aporía y muestra una de las formas más elaboradas de la fenomenología. Aquí la historia 
del motivo implica la posibilidad de una regresión (Rückfrage) trascendental a través de una historia inteligible y transparente a la conciencia, una historia cuyas sedimentaciones se puedan deshacer y rehacer sin alteración. Se pone en evidencia una nueva concepción de la intencionalidad dirigida a la historia y a la teleología. Este nuevo análisis histórico-intencional de la conciencia tiene como objetivo reactivar el sentido originario de los actos o de los productos históricos de la conciencia. Esto nos permite volver a trazar la génesis intencional de la geometría y definir mediante este ejemplo, el tipo de análisis por el que es posible siempre volver a captar en su nacimiento mismo, la originalidad trascendental de una producción histórica de la conciencia (Derrida, 1990, p. 260).

Husserl quiere volver al tema del origen y al fundamento de toda idealidad que aparece en la historia. Volviendo así a los problemas que despertaron sus primeras interrogantes filosóficas: la génesis empírica. Para ello invoca como respuesta una naturaleza eterna del hombre que se comprende y se reconoce como animal racional, es decir, como una razón que se oculta y se devela en la historia. Esto último hace pensar que aunque Husserl esté resuelto a centrarse en esta relación entre idealidad e historicidad, no alcanza la fundación intencional que haría posible una filosofía pura de la historia (Derrida, 1990, p. 271).

En 1954, el joven Derrida no entiende esta incapacidad como una debilidad de la filosofía de Husserl; por el contrario, asume que la imposibilidad de una aproximación absoluta al fundamento originario ante-predicativo y no constituido responde a la vocación propia de la fenomenología y de toda auténtica filosofía, cuyos fundamentos y respuestas deben ser replanteados continuamente. El propio Husserl lo reconoce en sus últimos días y confiesa haber dejado su tarea inacabada. De este modo, la realización está siempre por venir y expresa una necesidad intrínseca de un nuevo comienzo para alcanzar nuevas respuesta (Derrida, 1990, p. 283). Esta tarea de nuevo comienzo de la filosofía será el espíritu que anime la actividad fenomenológica de Derrida expuesta en los escritos que siguen a su Memoria de 1954. A continuación, intentaremos presentar el problema de la teleología en la fenomenología derridiana.

\section{LA VÍA TELEOLOGÍA: ENTRE GÉNESIS Y ESTRUCTURA}

La segunda incursión fenomenológica de Derrida se llevará a cabo durante los días 25 de julio y 3 de agosto de 1959 en el Centro cultural de Cerisy-La-Salle por ocasión de las “Conversaciones sobre la noción de «génesis» y de «estructura» bajo la dirección de Maucice de Gandillac, Lucien Goldmann y Jean Piaget. ${ }^{4}$ Años

\footnotetext{
${ }^{4}$ Las actas que recogen las conferencias y las discusiones que les siguieron se publicaron en 1965 (Gandillac, Goldmann, \& Piaget, 1965).
} 
más tarde Derrida recordará que Piaget solía llamarlo durante este congreso "el fenomenólogo" (Derrida, 2005, p. 258). La conferencia de Derrida lleva por título «Génesis y estructura» y la fenomenología. ${ }^{5}$ La tesis fundamental que defiende a lo largo de su presentación es que la fenomenología es una filosofía de la apertura en oposición a la metafísica de la clausura y al explicativismo de las ciencias empíricas. Para Derrida el fenomenólogo es el verdadero positivista que vuelve a las cosas mismas y desaparece ante la originalidad y la originariedad de las significaciones. Por tanto, Husserl al rechazar todo sistema de la clausura especulativa, se muestra más atento a la historicidad del sentido, a la posibilidad de su llegar a ser, respeta más lo que en la estructura permanece abierto (Derrida, 1965, pp. 243-244).

Derrida retoma la idea con la que concluye su Memoria: la auto-exigencia de profundización que la fenomenología va explicitando en cada progreso. De este modo, la filosofía de Husserl afronta un debate que regula y marca el ritmo de la marcha de la descripción, que le presta su "animación” y cuyo "inacabamiento", al dejar en desequilibrio cada gran etapa de la fenomenología, hace indefinidamente necesarias una reducción y una explicitación nuevas. Al mismo tiempo, este debate parece obligar a Husserl a transgredir el espacio puramente descriptivo y la pretensión trascendental de su investigación, hacia una metafísica de la historia, en la que la estructura sólida de un telos le permitiría reapropiarse -esencializándola y prescribiéndole de alguna manera el horizonte- de una génesis salvaje que se convertía cada vez más en invasora y que parecía corresponderse cada vez menos con el apriorismo fenomenológico y con el idealismo trascendental (Derrida, 1965, pp. 245-246).

La fenomenología es, según explica Derrida, el último estadio de diferenciación de una serie de etapas que van desde la cultura pre-teorética a la del proyecto teorético o filosófico (Derrida, 1965, p. 258). Derrida ve en la fenomenología la respuesta a la necesidad de la dialéctica de la historia universal. Solo en ella se da la presencia del telos en la consciencia fenomenológica como anticipación teorética infinita que se ofrece simultáneamente como tarea práctica infinita. Esta se da en la evidencia fenomenológica como evidencia de un desbordamiento esencial de la evidencia actual y adecuada. Por tanto, el proyecto mismo de la fenomenología se realiza desbordando esencialmente su sistema de evidencias o de determinaciones actuales y lo desborda como su fuente o su fin (Derrida, 1965, p. 259).

Sobre la cuestión del problema de la estructura y el problema de la génesis en la fenomenología, Derrida sostiene que Husserl intentaba conciliar la exigencia estructuralista que conduce a la descripción comprensiva de una totalidad, de

\footnotetext{
${ }^{5}$ Conferencia del 31 julio de 1959. El texto definitivo se publicó con las actas de las Conversaciones e incluye la discusión seguida de la conferencia (Derrida, 1965). Reeditado en (Derrida, 1967a, pp. 229-251).
} 
una forma o de una función organizada según una legalidad interna en la que los elementos solo tienen sentido en la solidaridad de su correlación o de su oposición, con la existencia genetista, es decir, el requerimiento del origen y del fundamento de la estructura (Derrida, 1965, p. 246). Derrida explica que la más ingenua puesta en práctica de la noción de génesis y sobre todo de la noción de estructura, supone al menos una delimitación rigurosa de las regiones naturales y de los dominios de la objetividad y esta delimitación previa, esta dilucidación del sentido de cada estructura regional depende necesariamente de una crítica fenomenológica (Derrida, 1965, p. 249). Esto explicaría que antes de tratar el problema de génesis, Husserl haya tenido que aterrizar primero en una fenomenología estática. Sin embargo, el problema de la génesis es preguntar acerca del sentido de la noción de estructura o de génesis en general, antes de las disociaciones introducidas por la reducción, es preguntar acerca de lo que precede a la reducción trascendental (Derrida, 1965, p. 259).

Esta tarea la lleva a cabo Husserl en su fenomenología genética posterior a Ideas $I$. La profunda unidad de esta descripción genética se difracta, sin dispersarse, según tres direcciones: la vía lógica-Experiencia y juicio y Lógica formal y lógica trascendental-, la vía egológica -Meditaciones cartesianas- y la vía históricoteleológica -Crisis y El origen de la geometría (Derrida, 1965, pp. 256-258). Esta tercera vía es la que deberá dar acceso al eidos de la historicidad en general, es decir, a su telos, pues el eidos de una historicidad, y así, del movimiento del sentido, movimiento necesariamente racional y espiritual, solo puede ser una norma, un valor más que una esencia. Por tanto, esta vía no es una vía entre otras, sino que es el momento en el que Husserl se enfrenta directamente con el problema de la génesis.

A partir de la vía teleológica, Derrida realiza una interesante explicación sobre la relación entre logos, historia y ser. En primer lugar, la irrupción del logos es el advenimiento a la consciencia humana de la idea de una tarea infinita de la razón (Derrida, 1965, p. 257). Para Husserl esta razón que se desvela a sí misma es el logos que se produce en la historia. Sale de sí para recogerse en sí, en el presente viviente de su presencia ante sí mismo. Este logos que se invoca y se interpela él mismo como telos y cuya $\delta \dot{v} v \alpha \mu \iota$ tiende hacia su $\varepsilon \dot{v} \varepsilon \dot{\rho} \rho \varepsilon \imath \alpha$ o su $\varepsilon \dot{v} \tau \varepsilon \lambda \varepsilon^{\prime} \chi \varepsilon l \alpha$ (Derrida, 1965 , p. 258). Por tanto, el logos no es nada fuera de la historia y del ser, porque es discurso o discursividad infinita y no infinitud actual porque es sentido. Si se plantea la cuestión a la inversa, ninguna historia como tradición de sí y ningún ser tendrían sentido sin el logos, que es el sentido proyectándose y profiriéndose a sí mismo (Derrida, 1965, p. 258). Derrida ve este logos como un telos completamente abierto o como la apertura misma. Por tanto, decir que es el más potente a priori estructural de la historicidad no es designarlo como un valor estático y determinado que daría forma y encerraría a la génesis del ser y del sentido. Por el contrario, es la 
posibilidad concreta, el nacimiento mismo de la historia y el sentido del devenir en general. En otras palabras, es estructuralmente la génesis misma en tanto origen y como llegar a ser (Derrida, 1965, p. 259).

El logos será telos, arché y $\delta$ v́vals. Según vemos, la teleología empieza a convertirse en la historia racional del ser en el mundo. Algunos años más tarde, en una reseña hecha a Lebenswelt und Geschichte de Hubert Hohl vinculará el concepto fenomenológico de intencionalidad, con el de teleología y añadirá:

"esta no es más que una teleología filosófica, lo que Husserl en un fragmento llama «el camino no confesional hacia Dios». En un sentido en el que lo insólito permanece por determinar -y por preservar-, es una Fe que el Saber habría reconocido como su propia condición. Tan es verdad que «dios ha acaecido siempre en la filosofía e incluso en la metafísica de la vida y de su mundo histórico»” (Derrida, 1963).

Vemos ya como Derrida empieza a establecer una relación profunda entre telos, logos y Dios. En las páginas que siguen intentaremos comprender esta nueva propuesta de la fenomenología del joven Derrida.

\section{El SENTIDO TEO-TELEOLÓGICO DE LA FENOMENOLOGÍA}

Derrida en su Introducción a la traducción francesa de El origen de la geometría de Husserl, a partir de la tematización de la posibilidad de la historia de la ciencia $-\mathrm{y}$ en concreto el origen de la geometría-, nos conduce al sentido de la historia en general (Derrida, 1962, p. 5). En los escritos anteriores, según hemos visto, el vínculo entre telos y logos se hacía explícito. Ahora, teniendo como propósito la sistematización de una fenomenología de la escritura y del documento, realiza un tránsito del logos al problema del lenguaje en general (logos como palabra). En este sentido, el telos se expresa en la historia mediante una humanidad entendida como comunidad (trascendental) de lenguaje (Derrida, 1962, p. 74). E1 telos sería, entonces, un horizonte de lenguaje universal en el que la humanidad (re) encontraría lingüísticamente el eidos como realización histórica. Esta universalidad sería, al mismo tiempo, la condición apriorística y teleológica de toda historicidad (Derrida, 1962, p. 107). Además, Derrida asume que en este último escrito de Husserl la recuperación total de los orígenes es un horizonte teleológico (Derrida, 1962, p. 108).

El tema de la teleología sirve de medio para profundizar la noción de apertura infinita, ya expuesta por Derrida con bastante rigor, y que es una de las señas de identidad de su propia fenomenología. En esta concepción se muestra que el sentido teleológico y el sentido de origen siempre se implican (Derrida, 1962, p. 141), pues anunciándose el uno al otro, no se revelan plenamente sino el uno por el otro, en el polo infinito de la historia (Derrida, 1962, p. 142). Visto así, la apertura del 
infinito es la apertura de la historia misma, en la mayor profundidad y en la mayor pureza de su esencia (Derrida, 1962, p. 143). A partir de esta apertura infinita un horizonte infinito se presenta como siempre posible. El horizonte es el siempre-ya ahí de un futuro al que preserva intacto, aun cuando se ha anunciado a la conciencia la indeterminación de su apertura infinita (Derrida, 1962, p. 123). Si el horizonte está inscrito desde el principio, este funciona como una determinación estructural de toda indeterminación material, que está siempre virtualmente presente a toda experiencia de la que es a la vez unidad e inacabamiento, unidad anticipada en todo inacabamiento. Por tanto, el horizonte hace coincidir lo a priori y teleológico (Derrida, 1962, p. 123).

Vemos, entonces, cómo la noción de telos va asumiendo nuevas relaciones y concretizaciones en el interior del proyecto fenomenológico. La fenomenología será, por tanto, teleológica (sentido) y arqueológica (génesis), dos aproximaciones que para Derrida están siempre co-implicadas (Derrida, 1962, p. 141). Sin embargo, esta no es la única novedad de la exposición del joven filósofo, pues añadirá que el telos de la geometría -o de cualquier ciencia- está siempre circunscrito en el marco de un Telos universal que "lo atraviesa, lo precede y lo supera" (Derrida, 1962, p. 142). Ahora bien, la fenomenología como intención final de la filosofía -y se podría decir del saber en general- realizaría el sentido mismo de la historia, es decir, sería el cumplimiento de esta teleología universal de la razón (Derrida, 1962, p. 155).

Es aquí, precisamente, cuando el tema del telos asume una nueva modalidad: una divinidad trascendental (Derrida, 1962, p. 163). Al parecer, Husserl sitúa este logos absoluto más allá de la subjetividad trascendental (Derrida, 1962, p. 162). Al asumir esta problemática, Derrida se vuelca sobre los Manuscritos ya clásicos en los que Husserl se refiere a Dios (E III) como polo ideal. Derrida intenta superar la aparente ambigüedad que se ha venido repitiendo desde la publicación de Ideas I, sobre la relación del Absoluto trascendental como divinidad con el Absoluto trascendental como subjetividad histórica (Derrida, 1962, p. 163).

"La conciencia divina, reveladora de la intangibilidad de las esencias constituidas, es, en tanto contenido de una ficción y como el Telos ordenador del universo real, una facticidad. La reducción de Dios como ser y conciencia fácticas libera la significación de una divinidad trascendental, tal como aparece en los últimos escritos" (Derrida, 1962, p. 163).

Y continúa,

"en ocasiones el Logos se expresa a sí mismo a través de una historia trascendental, y en ocasiones no es sino la autenticidad polar absoluta de la historicidad trascendental misma" (Derrida, 1962, p. 163). 
Vemos ahora que el telos como divinidad trascendental es también el logos como razón que se realiza en la historia. Derrida ve en esto el gesto mediante el cual Husserl intenta superar la metafísica mediante el uso de los mismos términos de la tradición metafísica (Derrida, 1962, p. 164). Esta idea expresada por Husserl desde Meditaciones cartesianas pone en evidencia que el joven Derrida considera que el padre de la fenomenología es un revolucionario, pero su revolución -como lo es toda revolución- pasa por una lucha en el interior del sistema. Se podría decir que el gesto mismo de aquello que Derrida denominará más adelante "deconstrucción" puede ya verse en el mismo Husserl e incluso podríamos decir -arriesgándose con seguridad a las inmediatas críticas de los derridianos ortodoxos- que este gesto es fruto también de la influencia de una cierta forma de leer e interpretar a Husserl.

Esta divinidad es, para Derrida, tanto aquello mediante lo que el sentido de la historicidad trascendental se da, como el Logos que está en el origen de la historia. Esto quiere decir que Dios sería el telos y el arche, el principio y el fin de la historia. Por esta razón, es también "el cumplimiento final y situado en el infinito, el nombre del horizonte de los horizontes y la Entelequia de la historicidad trascendental misma" (Derrida, 1962, p. 164). Esta divinidad como polo, que se sitúa "más allá", lo está solo en un sentido, pues también está implicada en la historia misma como lugar de realización de todo eidos y de toda idealidad. Por ello, la "diahistoricidad o metahistoricidad del Logos divino no atraviesa y no supera más que el Hecho como ya-hecho de la historia y simplemente sería el movimiento puro de su historicidad" (Derrida, 1962, pp. 164-165).

En un ingenioso intento de recuperar la idea anterior de logos como lenguaje o, más concretamente como "palabra", Derrida busca vincular la divinidad trascendental como palabra o habla (parole) en la historia como movimiento (Derrida, 1962, p. 166). Después de esta reflexión y corrección a los problemas de la ambigüedad sobre la noción de Dios en los manuscritos, Derrida intentará mostrar como este "habla" en tanto "hecho histórico" es una exigencia del ser en la historia. Vemos aquí empezar la introducirse en un lenguaje y reflexión que lo acercan a las propuestas de Heidegger sobre la historia del ser. ${ }^{6}$ El problema de Dios se integrará dentro de esta nueva perspectiva. Se podría interpretar que este ser que se hace Logos-palabra en la historia se identificará con el Telos-razón como horizonte de realización. En todo caso, el dios de Husserl así expuesto por el joven Derrida, sería un dios como horizonte de realización del sentido en la historia y no una persona.

\footnotetext{
${ }^{6}$ En el artículo La fenomenología y la clausura de la metafísica de 1966, Derrida repite muchas de las ideas señaladas en la Introducción al "Origen de la geometría de Husserl". Por ejemplo, presente a Husserl como un revolucionario de la metafísica y lo equipara a Marx, Nietzsche o Heidegger (Derrida, 2018, pp. 168-169). Las últimas páginas de este artículo también intentan conciliar a Husserl y Heidegger mediante el recurso a la historia del ser (Derrida, 2018, pp. 184-185).
} 


\section{CONCLUSIÓN}

El joven Derrida como fenomenólogo nos ha presentado una rigurosa y documentada reflexión sobre la fenomenología de Husserl. Por un lado, toma distancia de los enfoques consagrados en Francia de los años 50 e intenta reconducir el debate sobre el sentido de la arquitectura conceptual husserliana. Por otro lado, se enfrenta a los críticos que buscan reducir la fenomenología o bien al idealismo o bien a la negación de la existencia. Por tanto, su propuesta parte del concepto de "génesis" para concluir en una revalorización del problema del ser en la historia desde la fenomenología. En este itinerario, Derrida muestra cómo la pregunta por la génesis y la pregunta por el sentido están inexorablemente conectadas. Telos y arche en el proyecto de Husserl de identifican, el primero como horizonte de significación y el segundo como eidos que se realiza en la historia en una apertura de posibilidad infinita.

En un segundo momento, y siempre desde la perspectiva de la fenomenología como filosofía de la apertura, Husserl introduce el problema del logos como razón y horizonte de sentido en la historia. La historicidad como temática fuerte en el segundo período de evolución de la fenomenología obliga a Derrida a plantear el problema de la tradicionalidad como medio de conservación del sentido (telos) en el mundo. Esto le lleva a considerar otro sentido del telos-logos como horizonte universal de lenguaje en el que la teleología coincidirá con una suerte de gramática pura que sería la condición de posibilidad de la tradicionalidad en general, de la cultura y de la historia.

En un tercer movimiento, Derrida muestra cómo en los manuscritos de Husserl el telos asume también la figura de una divinidad trascendental como sentido trascendental y trans-histórico. Asimismo, afirma que esta divinidad no es un simple polo opuesto a la subjetividad trascendental, sino que también encuentra su realización en la historia como parole. Por último, el joven Derrida nos ha presentado una vía teológica de la historicidad y del sentido que inevitablemente se encuentra con el problema de Dios. Aunque su respuesta no deja de moverse en un plano abstracto, sin entrar de lleno con los problemas de una religión positiva y mucho menos de una revelación concreta, el dios de la fenomenología del joven Derrida sería una idealidad en sentido estricto que poco o nada tendría que ver con la experiencia religiosa. 


\section{BIBLIOGRAFÍA}

Baring, Edward. (2011). The young Derrida and French philosophy, 1945-1968. Cambridge; New York: Cambridge University Press.

Bernet, Rudolf. (1986). Differenz und Anwesenheit. Derridas und Husserls Phänomenologie der Sprache, der Zeit,der Geschichte, der wissenschaftlichen Rationalität. Phänomenologische Forschungen, 18, pp. 51-112.

Bernet, Rudolf. (1990). Derrida et la voix de son maître. Revue Philosophique de la France et de l'Étranger, 180, 2, pp. 147-166.

Dastur, Françoise. (2016). Déconstruction et Phénoménologie. Derrida en débat avec Husserl et Heidegger. Paris: Hartmann.

Depraz, Natalie. (2000). De l'empirisme transcendantal: entre Husserl et Derrida. Alter: Revue de Phénoménologie, 8, pp. 52-68.

Derrida, Jacques. (1962). Introduction. Edmund Husserl. L'origine de la géométrie (pp. 3-171). Paris: Presses Universitaires de France.

Derrida, Jacques. (1963). Compte rendu de Lebenswelt und Geschichte. (Symposion. Philosophische Schriftenreihe.), Hubert Hohl. Les Études philosophiques, 18, 1, pp. 95-96.

Derrida, Jacques. (1965). «Genèse et structure» et la phénoménologie. In Maurice de Gandillac, Lucien Goldmann \& Jean Piaget (Eds.), Entretiens sur les notions de genèse et de structure, pp. 243-260. Paris: Mouton.

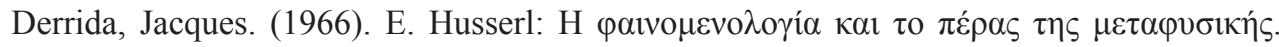

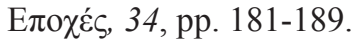

Derrida, Jacques. (1967a). L'Écriture et la différence. Paris: Éditions du Seuil.

Derrida, Jacques. (1967b). La voix et le phénomène. Introduction au problème du signe dans la phénoménologie de Husserl. Paris: Presses Universitaires de France.

Derrida, Jacques. (1990). Le problème de la genèse dans la philosophie de Husserl. Paris: Presses Universitaires de France.

Derrida, Jacques. (2000). La phénoménologie et la clôture de la métaphysique. Introduction à la pensée de Husserl. Alter: Revue de Phénoménologie, 8, pp. 69-84.

Derrida, Jacques. (2005). Le modèle philosophique d'une contre-institution. In Arlette Albert-Birot (Ed.), S.I.E.C.L.E, colloque de Cerisy : 100 ans de rencontres intellectuelles de Pontigny à Cerisy. Paris: Editions de l'IMEC.

Derrida, Jacques. (2013). Heidegger, la question de l'Être et l'Histoire cours de l'ENS-Ulm, 1964-1965. Paris: Éditions Galilée.

Derrida, Jacques. (2015). El problema de la génesis en la filosofía de Husserl (Javier Bassas Vila, Trans.). Salamanca: Sígueme.

Derrida, Jacques. (2018). La fenomenología y la clausura de la metafísica: introducción al pensamiento de Husserl (Traducción de Jimmy Hernández Marcelo). Investigaciones Fenomenológicas, 15, pp. 167-185. 
Ferraris, Maurizio. (2006). Introducción a Derrida. Buenos Aires: Amorrortu.

Ferraris, Maurizio. (2009). Documentalità: perché è necessario lasciar tracce. Roma: Laterza.

Ferraris, Maurizio. (2010). Ricostruire la decostruzione: cinque saggi a partire da Jacques Derrida. Milano: Bompiani.

Gandillac, Maurice de, Goldmann, Lucien, \& Piaget, Jean (Eds.). (1965). Entretiens sur les notions de genèse et de structure. Paris: Mouton.

Giovannangeli, Daniel. (2013). Husserl entre Tran Duc Thao et Derrida. Un moment de la phénoménologie en France. In Jocelyn Benoist \& Michel Espagne (Eds.), L'itinéraire de Tran Duc Thao: Phénoménologie et transfert culturel, pp. 133-146. Paris: Colin.

Héring, Jean. (1926). Phénoménologie et philosophie religieuse. Paris: Alcan.

Hernandez Marcelo, Jimmy. (2018a). El joven Derrida y la fenomenología francesa (19541967): fenomenología, epistemología y escritura. Mauritius: Editorial Académica Española.

Hernandez Marcelo, Jimmy. (2018b). Estudio introductorio a "La fenomenología y la clausura de la metafísica: introducción al pensamiento de Husserl". Derrida en la escuela de la fenomenología. Investigaciones Fenomenológicas, 15, pp. 135-165.

Lawlor, Leonard. (2002). Derrida and Husserl: The basic problem of phenomenology. Bloomington; Indianapolis: Indiana University Press.

Marrati-Guénoun, Paola. (1998). La Genèse et la trace: Derrida lecteur de Husserl et Heidegger. Dordrecht: Kluwer Academic Publishers.

Moati, Raoul. (2013). Phénoménologie et dialectique. Derrida critique de Tran Duc Thao. In Jocelyn Benoist \& Michel Espagne (Eds.), L'itinéraire de Tran Duc Thao: Phénoménologie et transfert culturel, pp. 147-162. Paris: Colin.

Peeters, Benoît. (2010). Derrida. Paris: Flammarion.

Perego, Vittorio. (2016). Derrida e la fenomenologia come epistéme. In Vittorio Perego (Ed.), Jacques Derrida. La fenomenologia e la chiusura della metafisica, pp. 5-41. Milano: La Scuola. 